\title{
An integrated NDE and FEM characterization of composite rotors
}

\author{
Ali Abdul-Aziz, ${ }^{*}$ George Y. Baaklini, ${ }^{\dagger}$ and Jeffrey J. Trude $1{ }^{\text {tb }}$ \\ ${ }^{a}$ Cleveland State University \\ ${ }^{b}$ National Aeronautics and Space Administration, Glenn Research Center
}

\begin{abstract}
A structural assessment by integrating finite-element methods (FEM) and a nondestructive evaluation (NDE) of two flywheel rotor assemblies is presented. Composite rotor $\mathrm{A}$ is pancake like with a solid hub design, and composite rotor $\mathrm{B}$ is cylindrical with a hollow hub design. Detailed analyses under combined centrifugal and interference-fit loading are performed. Two- and three-dimensional stress analyses and two-dimensional fracture mechanics analyses are conducted. A comparison of the structural analysis results obtained with those extracted via NDE findings is reported. Contact effects due to press-fit conditions are evaluated. Stress results generated from the finite-element analyses were corroborated with the analytical solution. Cracks due to rotational loading up to $48000 \mathrm{rpm}$ for rotor A and $34000 \mathrm{rpm}$ for rotor B were successfully imaged with NDE and predicted with FEM and fracture mechanics analyses. A procedure that extends current structural analysis to a life prediction tool is also defined.
\end{abstract}

Keywords: Finite element analysis, Computed topography, Stress analysis, Flywheels, Fracture mechanics, Nondestructive evaluation, FEM, NDE, Composites

\section{INTRODUCTION}

Composite flywheels are being developed as an alternative to expensive and short-life chemical batteries. Flywheels promise orders of magnitude increases in performance and service life in many NASA and military applications, including spacecraft, launch vehicles, aircraft power systems, uninterruptible power supplies, and planetary outposts and rovers.' Although the technology holds great promise, there remain a number of challenges to overcome, such as rotor certification for safe life, before these advanced flywheels reach operational status. Carbon-fiber-reinforced polymer composites are the materials of choice for energy applications because of the high energy and power densities that they can achieve. ${ }^{2}$ Flywheel design topology can also allow a burst failure mode that is relatively benign in comparison with flywheels made of metallic materials. ${ }^{3}$

A successful deployment of flywheels must address the long-term durability issue of polymer composites due to the limited availability of their fatigue characteristics and nonlinear behavior especially at elevated temperatures. NDE combined with structural analysis tools are being used to accurately assess the applicability of using various composite materials to design a suitable rotor/flywheel assembly. However, for NDE information to be useful in structural characterization and modeling, the NDE data format must be compatible with microstructural and structural models currently being developed. ${ }^{4}$

This paper describes the finite-element analyses and the NDE modality undertaken on two flywheel rotors that were spun to burst speed. Computed tomography (CT) and dimensional measurements were used to nondestructively evaluate the rotors before and/or after they were spun to the first crack detection. CT data findings of two- and three-dimensional crack formation were used to conduct finite-element and fracture mechanics analyses. A procedure to extend these analyses to estimate the life of these components is also outlined.

\footnotetext{
* Senior Research Scientist, Cleveland State University, Dept. of Civil and Env. Eng., NASA Glenn Research Center, 21000 Brookpark Rd., MS 6-1, Cleveland, Ohio 44135, Phone: 216-433-6729, Fax: 216-977-7150, Email: smazizagre.nasa.gov

${ }^{\dagger}$ Senior Research Scientist, Life Prediction Branch, NASA Glenn Research Center, 21000 Brookpark Rd., MS 6-1, Cleveland, Ohio 44135, Phone: 216-433-6016, Fax: 216-977-7150, Email: George. Y.Baaklini(agrc.nasa.gov

${ }^{\ddagger}$ Senior Aerospace Engineer, Structural Mechanics and Dynamics Branch. NASA Gilenn Research Center, 21000 Brookpark Rd., MS 49-8, Cleveland, Ohio 44135, Phone 216-433-5303, Fax 216-977-7051, Email: trudell(a)gre.nasa.gov
}

This is a preprint or reprint of a paper intended for presentation at a conference. Because changes may be made before formal publication, this is made available with the understanding that it will publication, this is maduced without the permission of the author. 


\subsection{Rotor test configurations}

Two spin-tested rotor configurations were analyzed and scanned. The first was rotor A, a single thick ring that was assembled on a solid aluminum hub. Rotor $\mathrm{B}$, the second configuration, is considered a mass-loaded device. The compressive radial stresses induced by the mass-loading hub help to overcome the poor transverse tensile strength of filament-wound composites; however, the disadvantage is that the circumferential and edge stresses at the inner diameter are increased. The following describes each layout.

1. Pancake rotor assembly $\mathrm{A}$ was assembled by U.S. Flywheel Systems, Inc." and the composite rim was manufactured by Toray Fiber, Inc." The rim inside diameter (id) is 6.9 in., the outside diameter is 11.5 in., and the height is $2 \mathrm{in}$. (fig. 1). The rim is made of $M 30 \mathrm{G}$ carbon fiber and an epoxy resin system. The radial interference fit between the rotor rim and the solid aluminum hub is 0.014 in.

2. Composite rotor assembly B was manufactured by U.S. Flywheel Systems and is shown in figure 2 . Rotor B has an approximate inside diameter of $8.9 \mathrm{in.}$, an outside diameter of $12.24 \mathrm{in.,}$ and a height of $8.5 \mathrm{in}$. The aluminum hub is designed to mass load the rim and is assembled with an interference fit to reduce radial tensile stresses and to prevent hub and rim separation. The rim is made of Hexel's IM7 carbon and AS4D carbon fiber and a shell epoxy resin mixture. The composite rotor rim is 78.3 percent fiber by weight and 71 percent by volume. IM7 fiber was wound at the inner diameter and outer diameter with AS4D fiber in the central portion. The radial interference fit between the composite rotor rim and the aluminum hub is $0.013 \mathrm{in}$.

\section{FINITE-ELEMENT ANALYSIS}

The loading state of a flywheel includes centrifugal, interference, thermal, and residual stresses. For axisymmetric rotors, analytical solutions are available to solve for the rotor stress state. ${ }^{5,6,7}$ The superimposed solutions assume plane stress and are only applicable for constant-axial-height, cylindrically orthotropic flywheels unless one uses numerical approaches. ${ }^{6 . x}$ Finiteelement analyses are performed when predictions of axial or shear stresses are needed, or if fracture mechanics analysis is to be performed. The ANSYS ${ }^{9}$ finite-element code was used to solve the nonlinear, two- and three-dimensional contact analysis, whereas the two-dimensional stress and fracture mechanics analyses were performed with MARC. "The material properties were generated using ICAN" because coupon test data were not available.

\subsection{Pancake Rotor A}

The MARC and ANSYS pancake rotor A models consisted of plane stress quad4 elements. The choice of the planar representation (plane stress) of a disk allows analysis of nonaxisymmetric rotors (i.e., bolt holes for hub-shaft attachment) and comparison with analytical solutions. An alternative approach would use axisymmetric elements to account for variations in the radial and hoop stresses as a function of axial height. In MARC, the nodes modeling the contact were intentionally offset in the hoop direction and were not radially aligned to accommodate the contact condition set by the MARC code. Using Manson's ${ }^{*}$ method modified by Genta ${ }^{6}$ coded in FORTRAN, the one-dimensional finite difference stress distribution of the rotor A was solved and compared with the two-dimensional solutions of MARC and ANSYS to benchmark the modeling approaches. ${ }^{12}$

\subsection{Rotor B}

The second mass-loaded hub was modeled in both two and three dimensions, which provided guidance to perform the fracture mechanics analysis in two dimensions. Both finite-element method (FEM) codes, ANSYS, and MARC were utilized to conduct these analyses. The rim stress distribution is clearly three dimensional due to the axial counterbore and its $1 / 8$ th cyclic symmetry, creating a nonuniform axial and circumferential mass loading of the hub. The three-dimensional model shown in figure 3 consisted of eight-node brick elements and the two-dimensional-model consisted of both quad4 and quad8 elements and is shown in figure 4 . The two reasons for using both quad4 and quad8 elements to model the rim were that (1) quad4 elements are needed to model the contact region between the hub and rim since higher order elements are not suitable for contact analysis because of a mismatch in stiffness between midside and comer nodes and (2) quad8 elements are required for subsequent fracture mechanics analysis. This however creates a region in which there is a stiffness mismatch between mid-side and corner nodes, although it is a region of low radial stress and not at the contact surface. Quarter symmetry was chosen to facilitate the application of boundary conditions to the $X, Y$-axes.

\footnotetext{
¿U.S. Flywheel Systems, Newberry Park, CA 91320

*Toray Hiber, Inc., Tacoma, WA 98446
} 


\section{FRACTURE MECHANICS}

The fracture mechanics analysis was invoked by activating the J-integral parametric option available in the MARC code. This resulted in the calculation of the change in strain energy due to nodal movement near the crack tip. Such quantity is evaluated by numerical integration at each increment for each prescribed nodal movement to determine the derivative of the strain energy with respect to the crack length at the end of each increment.

In addition to overcoming the difficulty that is usually encountered in the finite-element fracture mechanics representation of the solution near the crack tip, the mesh must be modeled so that the singularity is approximated with sufficient accuracy. Many methods have been established to arrive at such an approximation; however, the most commonly employed uses a degenerate form of the standard eight-node quadrilateral element. This method, usually referred to as the "1/4-point node technique," $" 2$ is also preferred for analyses conducted with the MARC code. It is applied by using an eight-node quarter of the edge length, the edge opposite the crack being kept straight. When these steps are followed, a singularity in the stress field exists in all directions going outward radially from the crack tip.

\subsection{Stress intensity factor}

The calculation of the stress intensity factor $K$ will allow one to determine at which load increment crack propagation occurs. This is done by calculating the elastic-plastic energy release rate, which is confined to a single parameter: the strength of the singularity in the elastic-plastic stress field at the crack tip. The magnitude of $K$ depends on the crack length, the distribution and intensity of applied loads, and the geometry of the structure. Crack propagation will occur when any combination of these factors causes $K$ to be equal to or greater than the experimentally determined material threshold value. ${ }^{13}$ The equation that relates $K$ to the energy release rate is ${ }^{9}$

$$
G=\frac{\mathrm{d} W}{\mathrm{~d} A}=\frac{K^{2}}{E}
$$

where $G$ is the energy release rate during a small crack extension; $W$ is the strain energy in pounds force per inch per inch; $K$ is the stress intensity factor in thousands of pounds per square inch (Ksi); A $=a \times b$ where $a$ and $b$ are the crack length and width in inches, respectively; and $E$ is the modulus in thousands of pounds per square inch (Ksi).

The stress intensity factor is given by

$$
K=\sqrt{E \frac{\mathrm{d} W}{\mathrm{~d} A}}
$$

After the functional forms of $K$ have been determined, the state of the stress and displacement near the crack tip region of the structure can be determined. It must be noted, however, that these equations represent a specific provision that describes the general approach to identifying these key parameters. Thus, for polymer matrix composites some modification will be required.

The life prediction computation is based on obtaining the data from the analysis and then applying the Paris relation, which ties the number of life cycles to the crack length and the stress intensity factor ${ }^{14}$ and is described by

$$
\mathrm{d} N=\frac{\mathrm{d} a}{c(\Delta K)^{n}}
$$

where $N$ is the number of loading cycles, $\mathrm{d} a$ is the incremental crack length, $c$ and $n$ are material constants determined from experimental data, and $\Delta K$ is the stress intensity factor range $\left(K_{\max }-K_{\min }\right)$. The loading cycle is defined as a combination of the press fit and the rotational speed loading.

Equations (1) to (3) identify a procedure that would lead to life assessment calculations. However, a fatigue crack growth database would have to be established for these materials to perform a life estimate.

\section{RESULTS AND DISCUSSION}

The results generated are presented in this section, which includes details covering each rotor assembly and the corresponding stress output. 


\subsection{Rotor A}

The radial stress distributions of the pancake rotor assembly A as predicted by the MARC code are shown in figures 5 and 6 for the entire rotor and for the rim section only. The peak radial stress of $3.4 \mathrm{Ksi}$ at $48000 \mathrm{rpm}$ occurs at a radial location of $4.35 \mathrm{in}$. as indicated in figure 6 . The peak rim hoop stress is $141 \mathrm{Ksi}$ at the $\mathrm{rim} / \mathrm{hub}$ interface. The radial and hoop stress distributions as calculated by ANSYS are shown in figures 7 and 8 . The peak radial stress is $3.52 \mathrm{Ksi}$ and the peak hoop stress $135.50 \mathrm{Ksi}$. The nodal peak radial stress occurred at a location of approximately 1.38 in. (nodal spacing was 0.115 in.) from the inside diameter of the $2.3 \mathrm{in}$-thick rim. The variation in radial stress versus radius for several speeds is shown in figure 9 . Note that the location of peak radial stress moves from the outer radius to the center as speed increases because the centrifugal loading continues to offset the compressive preload during assembly. Generally, carbon fiber exhibits an out-ofplane radial strength of 3 to $10 \mathrm{Ksi}$ and 200 to $300 \mathrm{Ksi}$ in the hoop direction. ${ }^{15}$

The results of Genta's finite difference method ${ }^{7}$ predicted a rim peak radial stress of $3.52 \mathrm{Ksi}$ at a radial location of $4.83 \mathrm{in}$., which is in excellent agreement with the ANSYS and MARC results as expected. The fiber volume ratio (FVR) was then reduced to 0.63 (but the same density of $0.69 \mathrm{FVR}$ was assumed) to show the sensitivity of reduced stiffness (due to translational inefficiencies, fatigue effects, variations in fiber and matrix properties) on the peak radial stress location. The peak radial stress increased to $3.52 \mathrm{Ksi}$ at the radial location of $4.38 \mathrm{in}$, a shift of 0.05 in. from the 0.69 -FVR results. The peak hoop stress decreased from $135.5 \mathrm{Ksi}$ to $126.9 \mathrm{Ksi}$. Finally, the residual stress was predicted based upon the cure cycle of the manufactured ring and superimposed with the centrifugal loading case. The peak radial stress with residual included increased to $6.40 \mathrm{Ksi}$ at the radial location of $4.67 \mathrm{in}$. The residual stress shifted the location of peak radial stress 0.15 in. versus 0.05 in. for a 7 -percent change in stiffness.

\subsection{NDE findings versus FEM of Rotor $A$}

After spin testing to $48000 \mathrm{rpm}$ (mass unbalance detected), CT images were taken to identify structural damage. Figure 10 shows a CT scan of the rotor after it was spun. An evaluation of the CT image indicated a cracking of the rotor along the entire circumferential direction. The crack is nearly symmetrical and is located at a range of 4.46 to 4.478 in. radial distance. The finite-element analysis reported in figures 6 and 7 showed that the maximum radial stress region is at a radial distance range of 4.35 to 5.34 in. Furthermore, the peak radial stress occurred at 4.78 in. or with the addition of estimated residual stress, at $4.67 \mathrm{in}$. With a 7 -percent stiffness reduction, the crack would move farther toward the center of the rim to 4.62 in. Because of the symmetrical nature of the crack, with accurate estimates or measurements of stiffness and residual stress and assuming no localized stress concentrations, the stress analysis can be correlated to crack location observed by the CT scan, which corroborates that the FEA is in good agreement with the NDE findings. The method requires an accurate determination of the material properties and residual stresses in the manufactured state to predict the peak radial stress and possible crack initiation site. In addition, comparing the predicted crack location with manufactured material properties and residual stresses versus NDE procedures provides an additional means of verifying and confirming service-related damage and design anomalies typically experienced by composite structural components.

\subsection{Mass-loaded hub Rotor B}

Figure 11 illustrates the two-dimensional radial stresses for Rotor B at its mid-plane at $34000 \mathrm{rpm}$, the speed at which a mass imbalance was detected. The stress distribution is the result of a complicated combined press-fit and centrifugal loading on a nonaxisymmetric structure. The peak rim hoop stress in the $2 \mathrm{D}$ model was $94 \mathrm{Ksi}$. A nonuniform radial stress distribution in the rim near the hub-rim interface developed because quad8 and quad4 elements were used in Marc, creating a mismatch in stiffness between the midside and comer nodes. The nonuniform stress distribution also created convergence problems and tremendous increases in CPU times. Figure 12 shows the three-dimensional hoop stress distribution generated in the flywheel at $34000 \mathrm{rpm}$. Note that the hoop stress reaches a maximum value of $113 \mathrm{Ksi}$ at the area of contact between the rim and the hub. The radial stress distribution at $34000 \mathrm{rmm}$ is shown in figure 13. Figure 14 shows the Von Mises stresses in the hub with a stress level of $32.4 \mathrm{Ksi}$ at the region adjacent to the holes, which indicates that the hub is very likely to fail at that particular section if a higher rotational load is applied.

Figure 15 illustrates the three-dimensional hoop stresses for the rim only at $34000 \mathrm{rpm}$. Note that a nonuniform hoop stress distribution is clearly shown. The maximum hoop stress occurs at the axial region below the counterbore of the hub, which can be explained by examining the radial stress distribution. Further, it is noted that high stress region is confined to a 1.92 in. height of the rotor, which coincides well with the crack initiation site reported by the CT scan shown in figure 22 . Figure 16 illustrates the three-dimensional radial stresses. Note the axial shift in radial stress due to the counterbore in the hub. The radial stress distribution due to combined residual, centrifugal, and interference loading is illustrated in figures 17 and 18 at $0^{\circ}$ and $22.5^{\circ}$. The superposition of the residual stresses creates a region of radial tensile stresses in the rim, while the interference fit and hub slots induce higher hoop stresses in the center than at the top of the rim. At $0^{\circ}$, the stresses decrease 
axially from the center to the top edge, with the peak radial stress occurring at a radial location of 5.48 to 5.68 in. At $22.5^{\circ}$, the peak radial stress location is nearly a constant 5.15 in. due to the slot cut in the hub.

The peak hoop stress distribution will be reversed as the speed is increased because the centrifugal forces decrease the initial interference preload near the center, and the counterbore mass-loading region of the hub induces higher compressive radial stresses. The mass loading of the counterbore section of the hub will also create higher bending stresses in the rim than that of a solid hub design, and shear stress levels will have to be addressed at the higher speeds. With regard to radial stress magnitude, the peak is $0.3 \mathrm{Ksi}$ without residual stress at a radial distance of $5.51 \mathrm{in}$ (fig. 16). In figure 17, the peak radial stress with residual is $1.3 \mathrm{Ksi}$ at $0^{\circ}$, with the peak radial stress at any given axial location varying from 5.48 to $5.68 \mathrm{in}$. in the radial direction. At $22.5^{\circ}$ (figure 17 ), the peak radial stress is $1.8 \mathrm{Ksi}, 0.5 \mathrm{Ksi}$ higher than the $0^{\circ}$ plane, with the peak radial stress at any given axial location nearly constant at $5.15 \mathrm{inch}$. Because of the slot in the hub at $22.5^{\circ}$, less radial compression is present resulting in a higher radial stress than the $0^{\circ}$ plane. The combined residual, assembly, and centrifugal radial stress state appears to be at the lower bound of the expected transverse strength levels and indicates a critical value that causes failure. Higher estimates of the residual stresses would shift the peak radial stresses inward toward the center of the rim, as would a decrease in hoop stiffness that was described in the previous section.

Figures 19 and 20 represent the two-dimensional radial stress distribution of the rotor obtained from the fracture mechanics analysis at a rotational speed of $34000 \mathrm{rpm}$. The crack was propagated at the nodal point of the element that exhibited the highest stress as indicated by an earlier three-dimensional stress analysis. An initial crack size of 0.005 in was assumed based upon both data suggested by the CT scan and visual inspection. Furthermore, the crack collected through the CT scan was asymmetric and it replicated the phenomena experienced by the flywheel while undergoing excessive centrifugal loading. In this work, however, to simplify the analytical calculations, the two-dimensional analyses assumed that the crack was being propagated symmetrically with respect to the radial direction and along the circumference of the flywheel.

Additional observations noted in viewing figure 20 are confined to the fact that the stress magnitude at the crack tip grew higher as the crack advanced. The counterbore in the hub is primarily responsible for the high rim bending stresses that contributed to the rotor cracking. Figure 21 presents the energy release rate versus the crack length. A highlighted linear variation (as anticipated) shows that the higher the energy rate, the higher the crack length. Furthermore, this denotes that a higher energy release rate would ultimately lead to unstable crack growth provided that the fracture toughness data of the material demonstrate such a possibility. This scenario will drive the crack to grow until it hits the edge or boundaries. Moreover, this information provides a preliminary guide for performing life analyses when fatigue crack growth data for these materials become available.

\subsection{NDE findings versus FEM of Rotor $B$}

The NDE-FEA results for rotor B are presented in this section. Figure 22 shows a computed CT scan for the rotor. The scan is a cross-sectional view taken in a direction parallel to the axis of rotation. The defects detected by the CT scan clearly show the two cracks, which vary in axial location because of the variation in the radial stress profile created by the mass-loaded hub and the rotational speed of $34000 \mathrm{rpm}$. The view indicates that the cracks are parallel to the rotor axis of rotation, they are asymmetrical along the entire radial direction, and they fall within 5.33 to 5.81 in at the middle section of the rotor. These data are in close agreement with the FE analysis, which initiated peak radial stresses at a radial distance of 5.15 in. at $22.5^{\circ}$ and between 5.48 and $5.68 \mathrm{in}$. at $0^{\circ}$. While the crack was asymmetrical along the mid-plane of the rotor as shown in figure 22 , the crack at the bottom of the figure closely follows the mirror image of the stress distribution shown in figure 17. It is speculated that the crack in the lower plane initiated first, and the resulting stress redistribution created the second crack. The variation of radial location is also affected by the circumferential changes in radial stress as shown in figures 16 and 19 . Both figures 17 and 18 clearly indicate that the maximum stress region is within the section defined by the CT scan.

Other supporting data relating NDE to FEA are seen in figures 18 and 22. For instance, the stress results shown in figure 18 , which represents the radial stresses in the rim, clearly indicate that the maximum stress region is within the section defined by the CT scan. Figure 18 describes the stress region at the contact side with the hollow hub whereas figure 22 shows the crack locations and variations within the rim. The measurements reported, as obtained from figure 22 , once more show that the NDE data correlate well with those predicted by the FEA.

Looking at the data collected from the CT scan and the FEA results allows us to conclude that with a good degree of accuracy, it was possible to detect the crack origination area and its direction. Furthermore, the NDE data assisted us in performing a three-dimensional, finite-element analysis to identify the crack and the maximum stress locations. Such information was greatly needed to grow a crack in the rim for life prediction purposes 


\section{CONCLUSIONS}

Two- and three-dimensional, finite-element stress analyses were conducted for two flywheel rotor systems with solid and hollow hubs, respectively. Subsequent two-dimensional fracture mechanics analysis were conducted to trace a crack propagation identified by computed tomography (CT) scans after spin loading to $34000 \mathrm{rpm}$. The results were discussed and compared with those predicted by CT. The following conclusions are drawn:

1. The analyses showed that the rotor first crack as detected by the CT scan could be modeled with finite elements provided that the material properties and residual loads are adequately quantified.

2. In-depth FEA modeling and evaluation of two flywheel rotor systems under spin loading conditions was successfully corroborated with NDE findings.

3. Crack location cited via the CT scan corresponded well with that simulated by the fracture mechanics analysis.

4. Lack of fatigue crack propagation data for the composite materials hindered conducting a life estimate as desired. Until a fatigue crack growth database is established, a life assessment cannot be completed.

5. Hub design is a crucial factor affecting the durability of the rotor because an improper hub design can result in high bending stresses that in turn result in complex crack propagation behavior. Therefore, the hub designer must try to minimize rim induced bending stresses.

\section{REFERENCES}

1. Asley, S.: Flywheels Put a New Spin on Electric Vehicles. Mech. Eng., vol. 115, no. 10, 1993, pp. 44-51.

2. Olszewski, M., et al.: On the Fly or Under Pressure. Mech. Eng., vol. 110, no. 6, 1988, pp. 50-58.

3. Coppa, A.P.: Flywheel Containment and Safety Considerations: An Assessment of Integrated Flywheel System Technology. NASA CP-2346, 1985, pp. 243-264.

4. Baaklini, G.Y., et al.: Structural Chatracterization of Metal Matrix Composites Using NDE Data. NASA CP-10192, Available from the Subsonic Systems Office, NASA Glenn Research Center, Carol A. Ginty.

5. Timoshenko, S.; and Goodier, J.N.: Theory of Elasticity. McGraw-Hill, New York, NY, 1970.

6. Genta, G.: Kinetic Energy Storage: Theory and Practice of Advanced Flywheel Systems. Butterworths, London, England, 1985.

7. Gabrys, C.W.: Design, Fabrication and Testing of Advanced Composite Energy Storage Flywheels (Filaments, Urethane Matrix). Ph. D. Dissertation, Pennsylvania State University, 1996.

8. Manson, S.S.: Determination of Elastic Stresses in Gas-Turbine Disks. NACA TN-1279, 1947.

9. ANSYS Finite Element Program. ANSYS Release 5.4, ANSYS, Inc., Canonsburg, PA, 1997.

10. MARC General Purposes Finite Element Analysis Program. Vol. A: User Information Manual; Vol. F: Theoretical Manual. MARC Analysis Research Corporation, Palo Alto, CA, 1996.

11. Murthy, P.L.N.; and Chamis, C.C.: Integrated Composite Analyzer (ICAN): Users and Programmers Manual. NASA TP-2515, 1986.

12. Abdul-Aziz, A.; Baaklini, G.; and Trudell, J.: "Structural Analysis of Composite Flywheels: An Integrated NDE and FEM Approach," NASATM-2001-210461, January 2001.

13. Nagtegaal, J.C.; and deJong, J.E.: Some Computational Aspects of Elastic-Plastic Large Strain Analysis. Int. J. Numer. Methods Eng., vol. 17, no. 1, 1981, pp. 15-41.

14. Rice, J.R.; and Tracy, D.M.: Computational Fracture Mechanics. Proceedings of the Symposium on Numerical and Computer Methods in Structural Mechanics, S.J. Fenves, et al., eds., Academic Press, NY, 1973, pp. 585-623.

15. Paris, P.; and Erdogan, F.: A Critical Analysis of Crack Propagation Laws. J. Basic Eng. Trans. ASME, vol. 85, 1963. pp. $528-534$.

16. Agarwal, B.D.; and Broutman, L.J.: Analysis and Performance of Fiber Composites. Second ed., John Wiley \& Sons, Inc., New York, NY, 1990. 


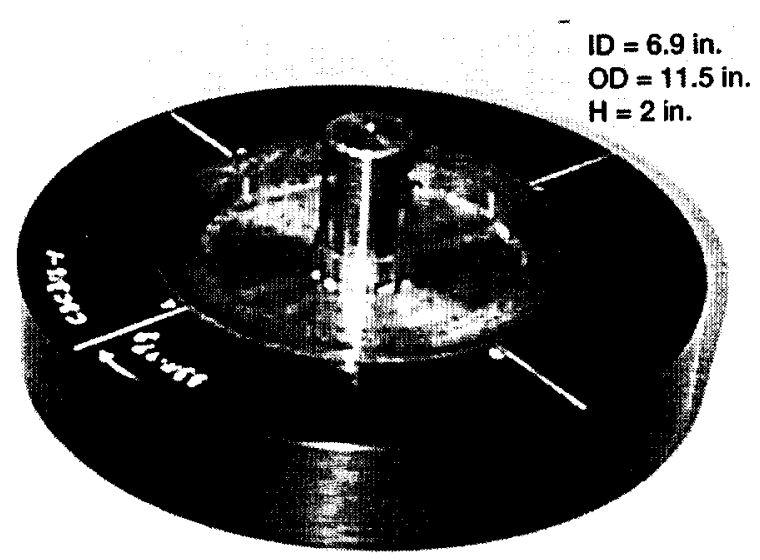

Figure 1.-Pancake rotor assembly $A$ with aluminum hub.

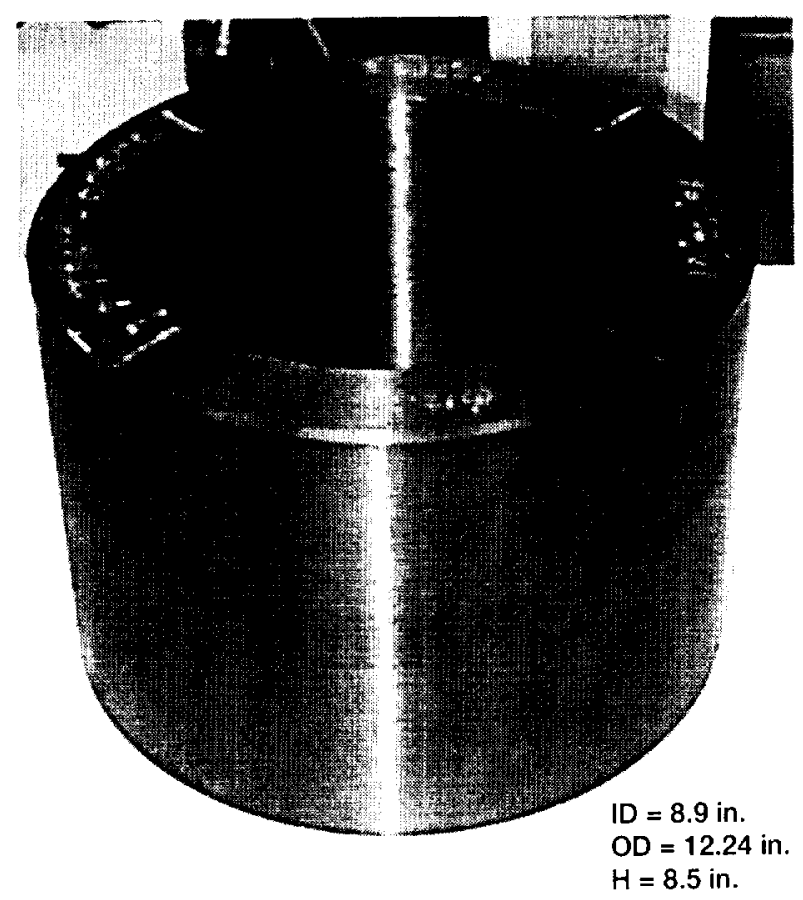

Figure 2.-Composite rotor assembly $\mathrm{B}$.

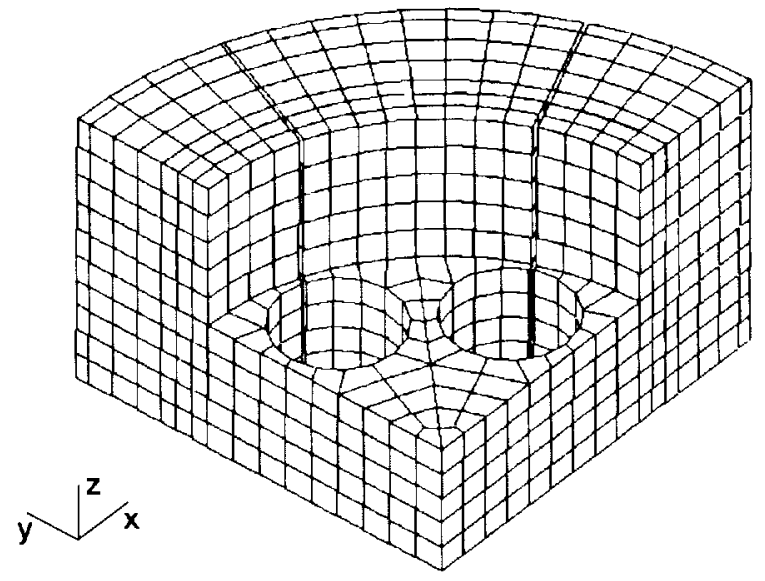

Figure 3.-Rotor B three-dimensional, finite-element model consisting of 1642 eight-node brick elements and 2283 nodes.

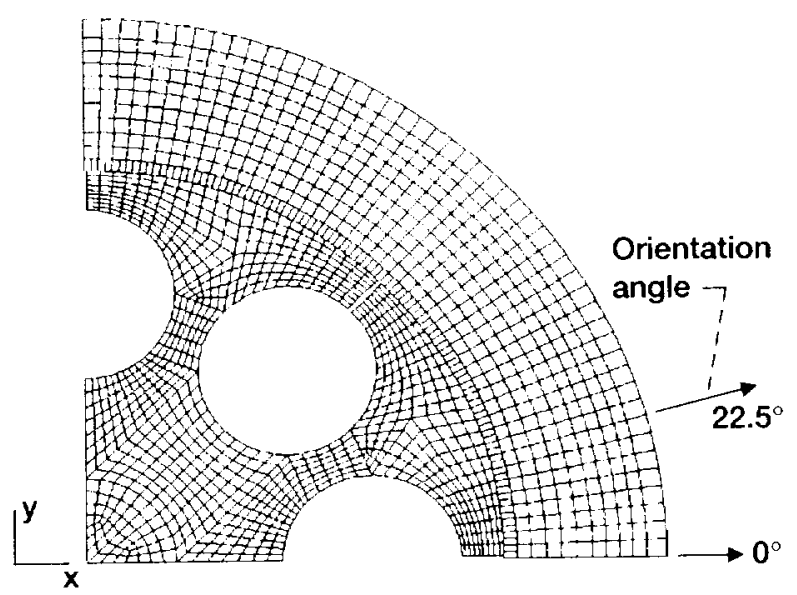

Figure 4.- Rotor B two-dimensional, finite-element model consisting of 1408 quad4 and quad8 elements and 2592 nodes. 


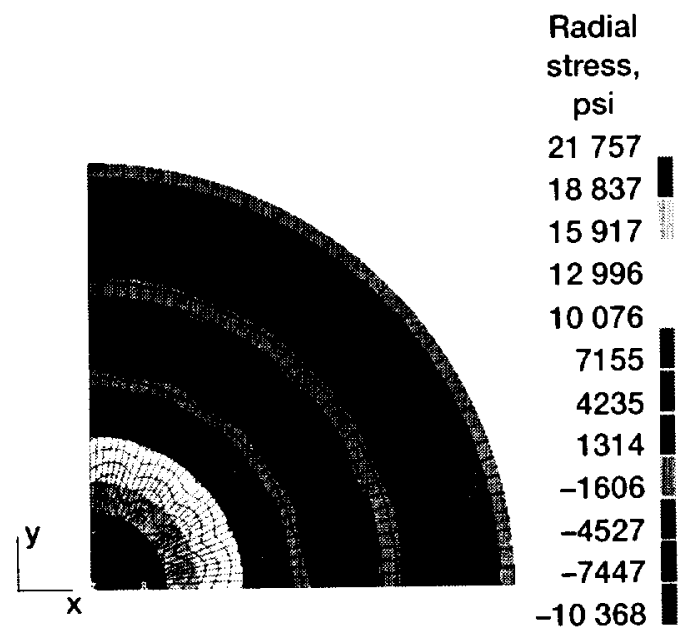

Figure 5.-Radial stress distribution for rotor A based on MARC analysis. Rotational speed, $48000 \mathrm{rpm}$.

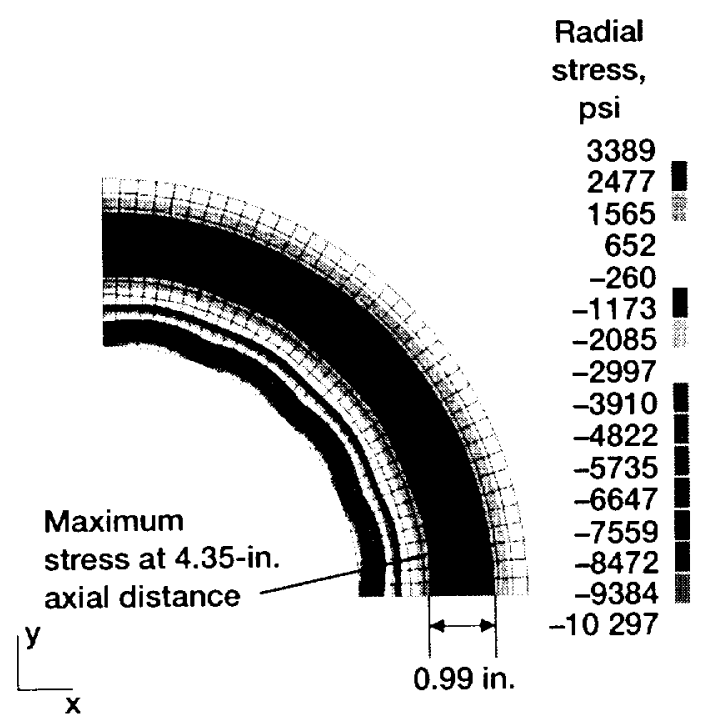

Figure 6.-Rim radial stress distribution for rotor A based on MARC analysis. Rotational speed, $48000 \mathrm{rpm}$.

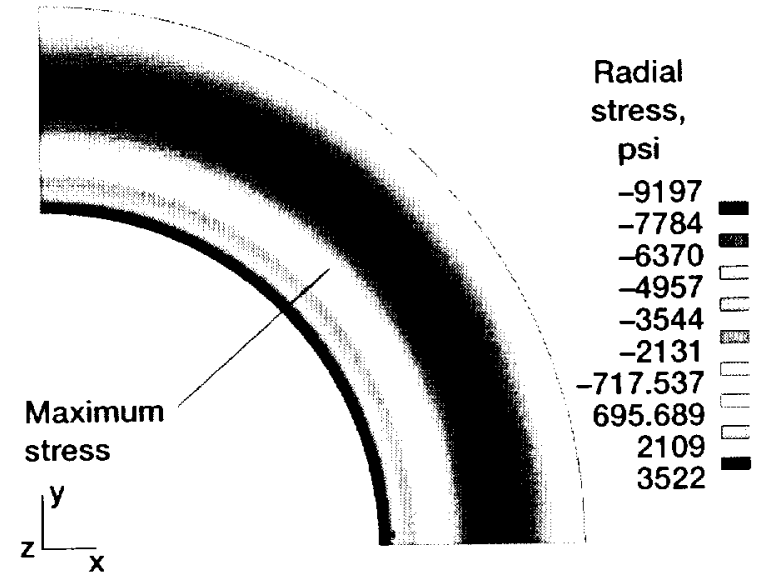

Figure 7.-Rim radial stress distribution for rotor A based on ANSYS analysis. Rotational speed, $48000 \mathrm{rpm}$.

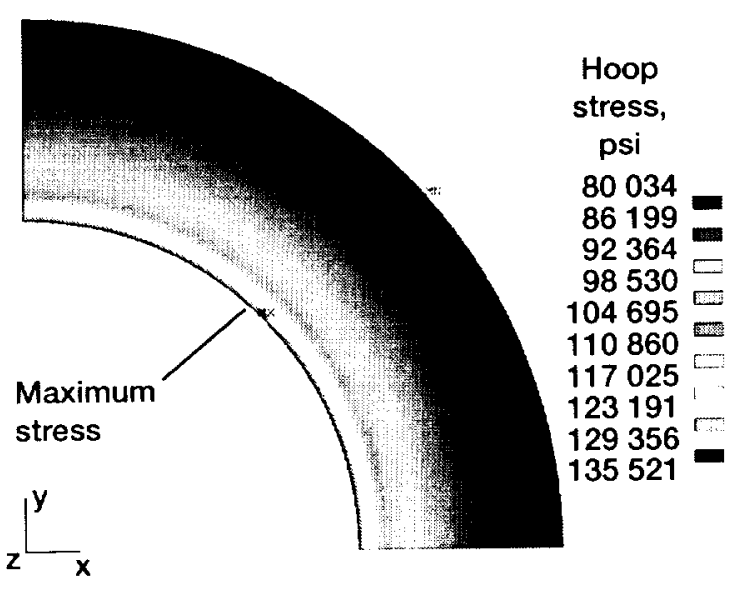

Figure 8.-Rim hoop stress distribution for rotor A based on ANSYS analysis. Rotational speed, $48000 \mathrm{rpm}$. 


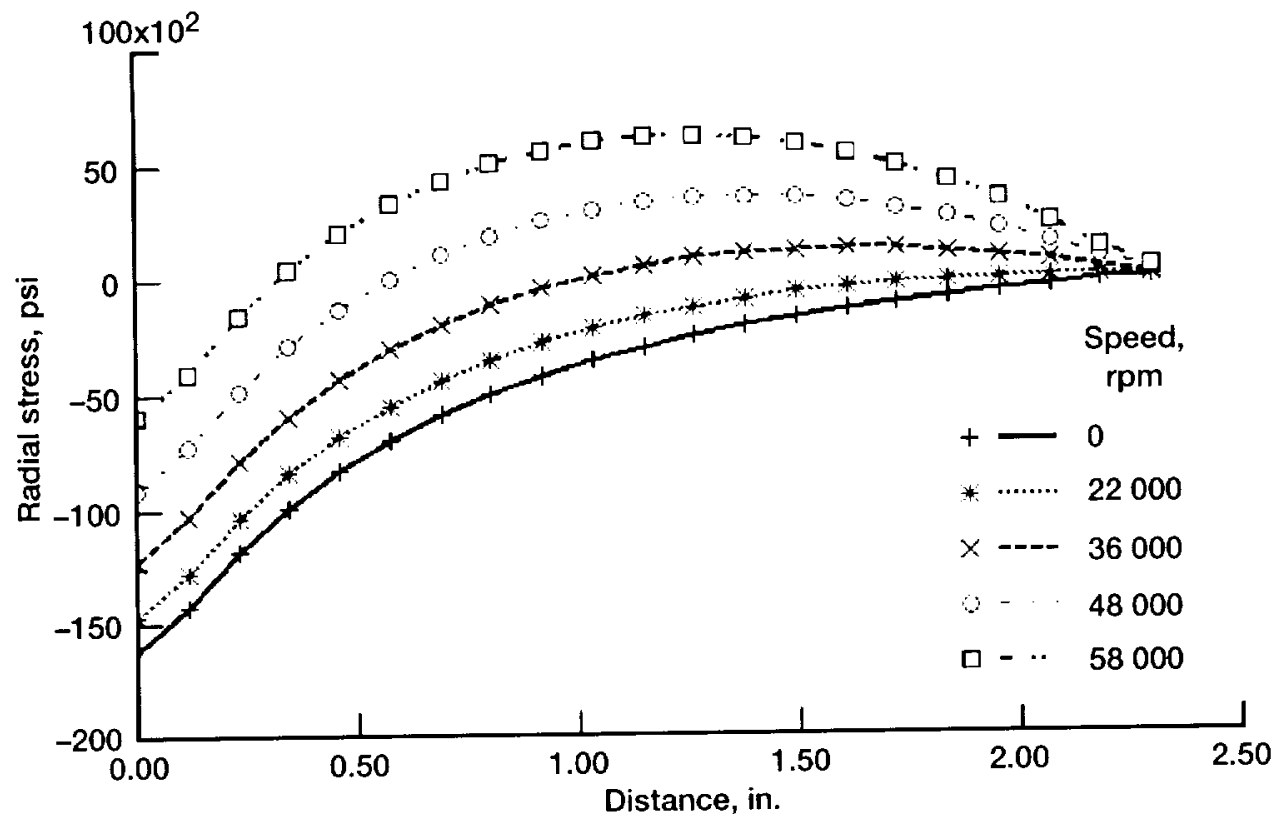

Figure 9.-Rim radial stress distribution as function of radius at several speeds for rotor A based on ANSYS analysis.

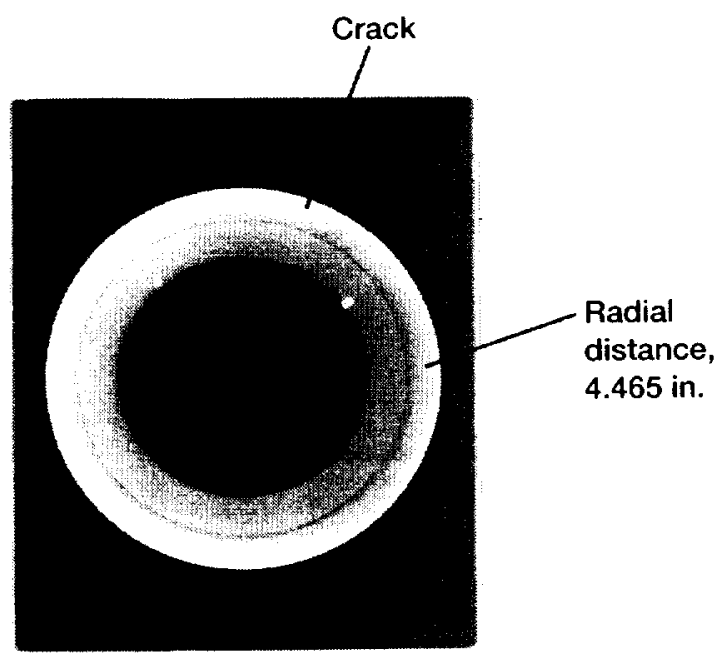

Figure 10.-Computed tomography scan of tested pancake rotor $A$.

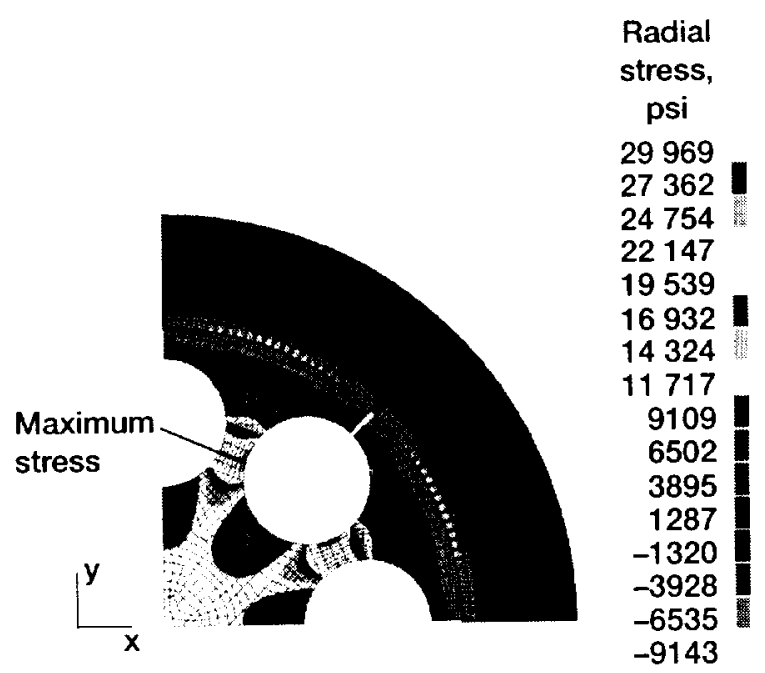

Figure 11.-Radial stress distribution for rotor B based on MARC two-dimensional analysis. Rotational speed, $34000 \mathrm{rpm}$. 


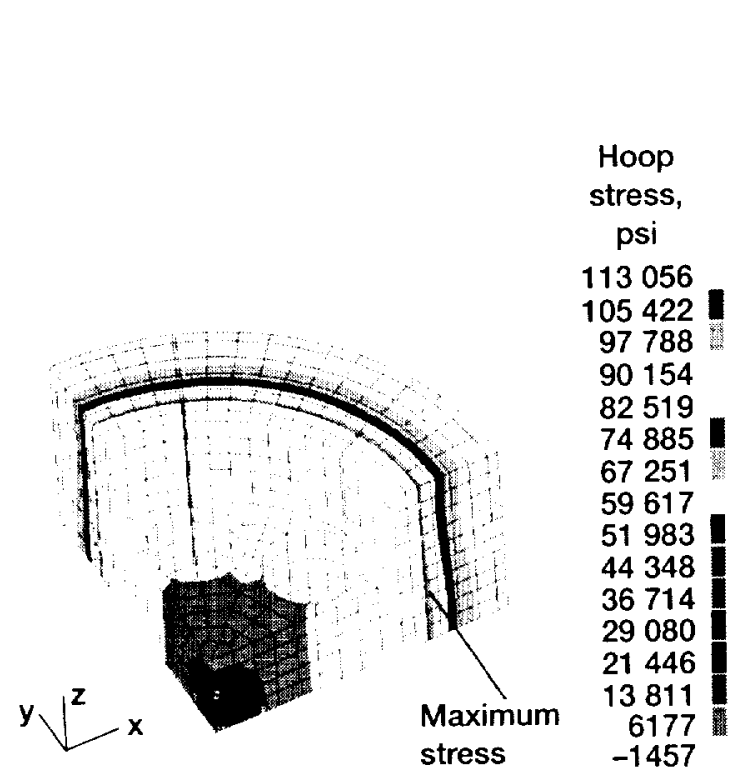

Figure 12.-Hoop stress distribution for rotor B based on ANSYS three-dimensional, finiteelement analysis. Rotational speed, 34000 rpm.
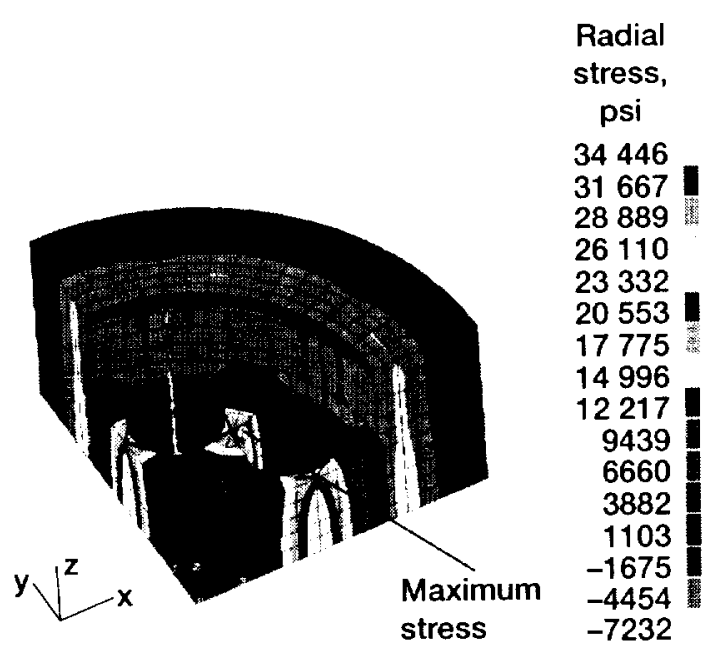

Figure 13.-Radial stress distribution for rotor B based on ANSYS three-dimensional, finiteelement analysis. Rotational speed, $34000 \mathrm{rpm}$.
Von Mises

stress,

psi

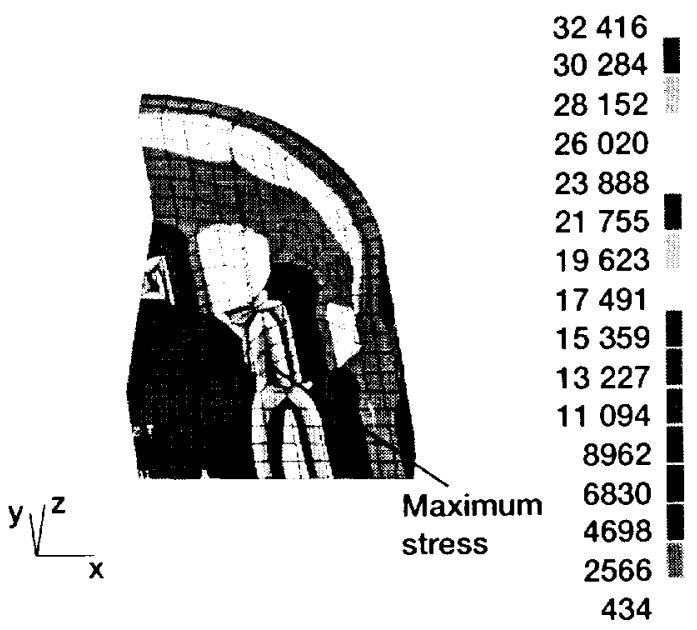

Figure 14.-Von Mises stress distribution for hub of rotor $B$ based on ANSYS threedimensional, finite-element analysis. Rotational speed, $34000 \mathrm{rpm}$.
Hoop

stress,

psi

113056

108464

103873

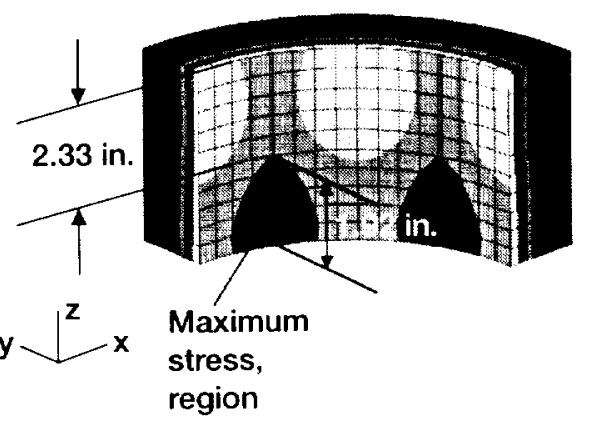

99281

94689

90097

85506

80914

76322

71730

67138

62547

Figure 15.-Rim hoop stress distribution for rotor $B$ based on ANSYS three-dimensional, finite-element analysis. Rotational speed, $34000 \mathrm{rpm}$. 



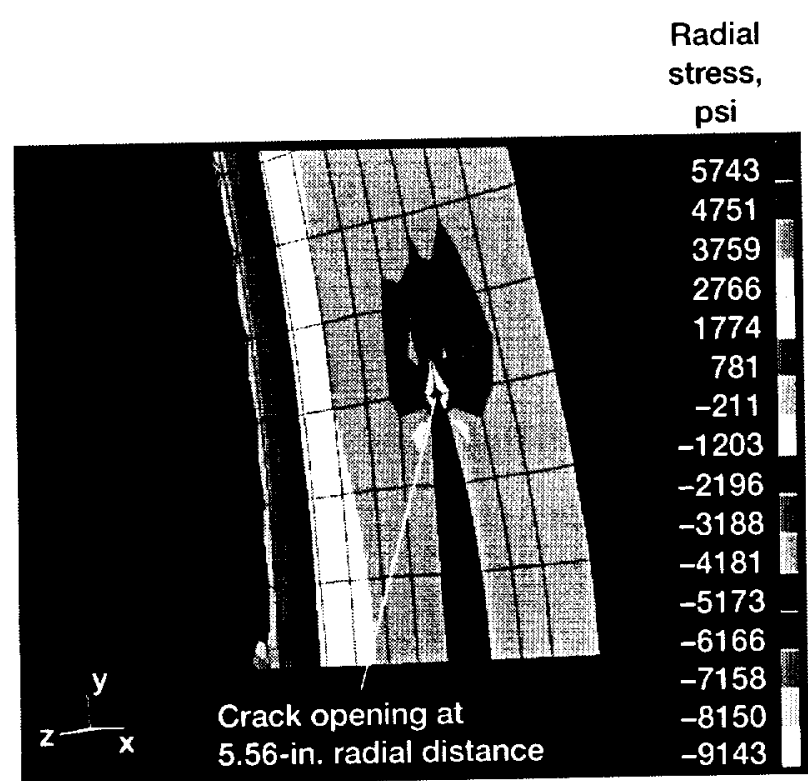

Figure 20.-Rim fracture mechanics radial stress distribution for rotor $B$ based on MARC analysis. Rotational speed, $34000 \mathrm{rpm}$ (closeup view of crack propagation).

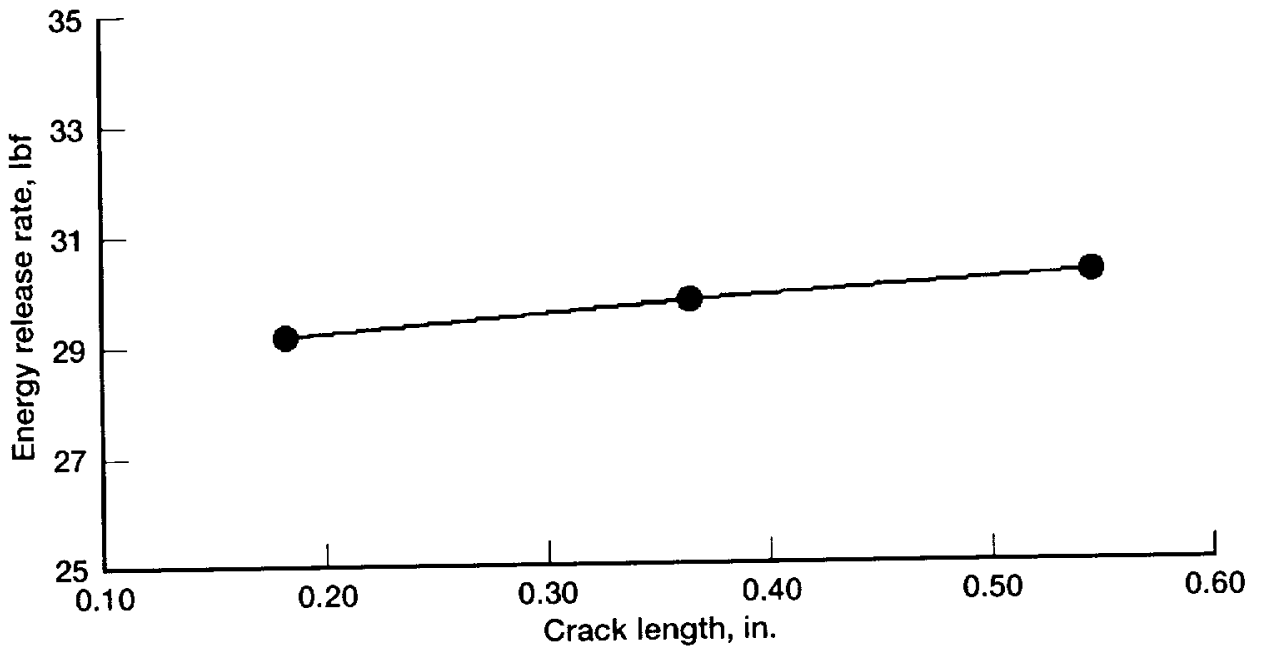

Figure 21.-Energy release rate as function of crack length for rotor $B$.

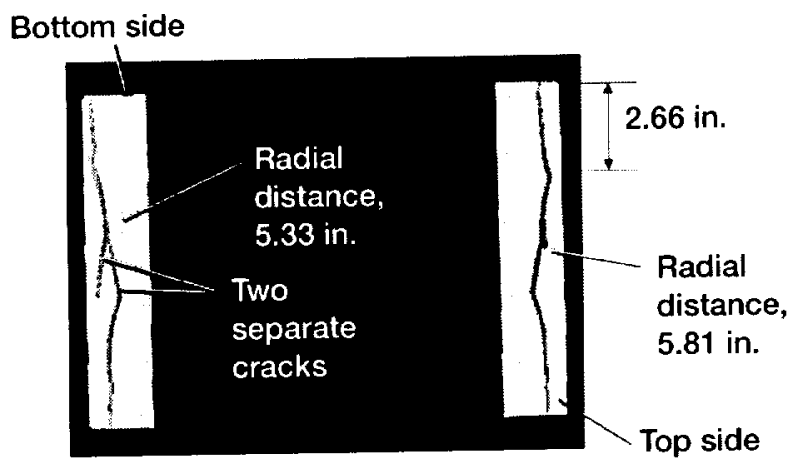

Figure 22.-Cross-sectional computed tomography scan of rotor $B$ showing cracking. 